showed significant improvements in social functioning, there were still obvious impairments within this population. Second, and very importantly, the differences between the cognitive therapy group and the control group were only apparent during the active phase of treatment - the control group continued to make modest gains during the follow-up period so that at 1 year after cognitive therapy there was no difference in social functioning between the two groups. One conclusion from this result is that individuals who receive 16 sessions of cognitive therapy for chronic or residual depressive symptoms may benefit from additional but less-frequent maintenance cognitive therapy sessions.

Lastly, Ito et al are right to point out that calculations of numbers needed to treat from this study are indeed indicative of substantial benefits from using cognitive therapy. For the record, using data from our study and other recent studies, only four to six additional patients need be treated with cognitive therapy to prevent one relapse.

J. Scott Department of Psychological Medicine Academic Centre, Gartnavel Royal Hospital, 1055 Great Western Road, Glasgow GI2 0XH

\section{Long-term psychotherapeutic relationships in schizophrenia}

I would like to add what I believe is an important factor not mentioned by Thornicroft \& Susser (2001) in their editorial on evidence-based psychotherapies in the community care of schizophrenia. It is a factor that I think is missing from a great deal of psychiatric literature on what helps patients get better and what makes us human. People with schizophrenia have withdrawn from being able to relate to others. They need somebody who is able to provide a long-term therapeutic relationship and is not frightened off by those who say 'beware of dependency' or seduced by the culture of brief interventions; a person who can stand up to the 'package culture' and stay with the patient and family over a long period of time. This sort of work does not make headlines. I think it is the role of psychodynamic psychotherapists to champion dependency in order that the patient can find something of his or her own from the shattered fragments of self; a mature dependence, within the constraints of illness. This work is not easy, requires support, supervision, time and resources. Perhaps the paucity of evidence is because this apparently simplistic viewpoint meets great resistance and is culturally dystonic.

Thornicroft, G. \& Susser, E. (200I) Evidence-based psychotherapeutic interventions in the community care of schizophrenia. British Journal of Psychiatry, 178, 2-4.

\section{Brogan Regional Department of} Psychotherapy, Claremont House, off Framlington Place, Newcastle upon Tyne NE2 4AA

\section{Psychodynamic thinking and the community mental health team}

I read with great interest Thornicroft \& Susser's (2001) editorial on evidence-based psychotherapeutic care in schizophrenia. It called for evidence-based interventions being implemented in the face of resource limitations and a remedy to the absence of implementation plans for well-established effective interventions such as family therapy. However, Thornicroft \& Susser dismiss psychodynamic approaches. Although the general view is that people with schizophrenia do not benefit from intensive psychoanalytic psychotherapy, there are some heroic efforts by analysts such as Herbert Rosenfeld (1987). In particular, such approaches do address the imperfection of our models of mental disorder.

One thing the psychodynamic way of thinking can offer members of the community mental health team (CMHT) is understanding of complex mental states from the patient's perspective, and new ways of understanding those that fall outside of our management strategies. There is no doubt that the delivery of psychoanalytic psychotherapy to people with schizophrenia, on an intensive basis, will not be resourced, nor will the symptom outcomes necessarily be better. Therefore, the cost cannot be justified. However, part of the problem that faces CMHTs is the long-term nature of their work with little reward in terms of symptom improvement and recovery for those with enduring severe mental illness. This can be frustrating and de-skilling for staff, particularly if they have a limited range of therapeutic models. I have worked in an assertive treatment team for the people with severe mental illnesses and one for homeless people with mental illnesses. Staff retain curiosity and capacity to think and question their formulations about patients in a psychodynamic way. Their work continues to be fresh and motivating. This is particularly welcome in light of Wykes et al's (1997) finding that CMHT staff are not uncommonly depersonalised and therefore unable to empathise with their patients. At a time when there is a movement to ensure good human relationships as well as therapeutic relationships with patients, dismissal of the relevance of psychodynamic thinking in the healthy functioning of a CMHT is premature. This is one area of CMHT functioning that warrants further research, as suggested by Thornicroft $\&$ Susser.

Rosenfeld, H. A. (1987) Impasse and Interpretation Therapeutic and Anti-Therapeutic Factors in the Psychoanalytic Treatment of Psychotic, Borderline and Neurotic Patients. London: Tavistock.

Thornicroft, G. \& Susser, E. (200I) Evidence-based psychotherapeutic interventions in the community care of schizophrenia. British Journal of Psychiatry, 178, 2-4.

Wykes, T., Stevens, W. \& Everitt, B. (1997) Stress in community care teams: will it affect the sustainability of community care? Social Psychiatry and Psychiatric Epidemiology, 7, 398-407.

K. Bhui Department of Psychiatry,

St Bartholomew's and The Royal London School of Medicine and Dentistry, Queen Mary \& Westfield College, University of London, Mile End Road, London El 4NS

\section{What came first: dimensions or categories?}

We read with much interest the paper by O’Dwyer \& Marks (2000), and think that the case vignettes reported by the authors fit perfectly with Insel \& Akiskal's (1986) model that considers obsessive-compulsive disorder as a disorder that can develop along a continuum of insight. Therefore, the primary problem is not the boundaries between obsessive-compulsive disorder (or anorexia, or body dysmorphic disorder) and psychosis, but rather at which point insight is lost and the disorder under consideration becomes a frankly psychotic one. If one considers insight as a dimension spanning from normality to the most severe psychiatric conditions, then it will not be difficult to posit several psychiatric disorders along it, with all possible heterogenous combinations. The model becomes even more comprehensive if we add the 'uncertainty/ 